\title{
Nontraumatic tube method for detecting a vessel responsible for colonic diverticular hemorrhage
}

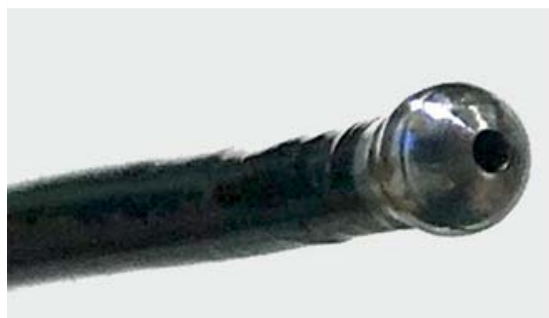

Fig. 1 Structure of a nontraumatic tube.

Colonic diverticular hemorrhage has been reported as the most common cause of lower gastrointestinal bleeding [1-3]. In colorectal diverticular hemorrhage, active bleeding, nonbleeding visible vessels, and adherent clot (indicating active bleeding or nonbleeding visible vessels when removed) are defined as stigmata of recent hemorrhage (SRH) [4]. For SRH identification, blood clot removal and detailed examination of the responsible diverticulum are needed. However, use of the scope function alone is sometimes challenging; thus, a simple and effective method that helps to identify the SRH more precisely is required.

A 65-year-old woman with hematochezia was admitted to our hospital. Colonoscopy revealed an adherent clot attached to the diverticulum in the ascending colon. The blood clot could not be removed by washing or suction using only an endoscope. Moreover, the diverticulum opening was too small to enable internal examination.

Therefore, we used a nontraumatic tube (Olympus, Tokyo, Japan) designed for the pit diagnosis of colonic tumors by magnifying endoscopy [5]. The tip of the nontraumatic tube is small and spherical and includes a spray hole ( $\mathbf{F i g} \mathbf{1}$ ). The tube can be inserted safely into the diverticulum owing to its shape. The blood clot can then be removed by repeated washing and suction via the spray hole (> Fig. 2).

After removing the blood clot in this way, we used the nontraumatic tube to expand

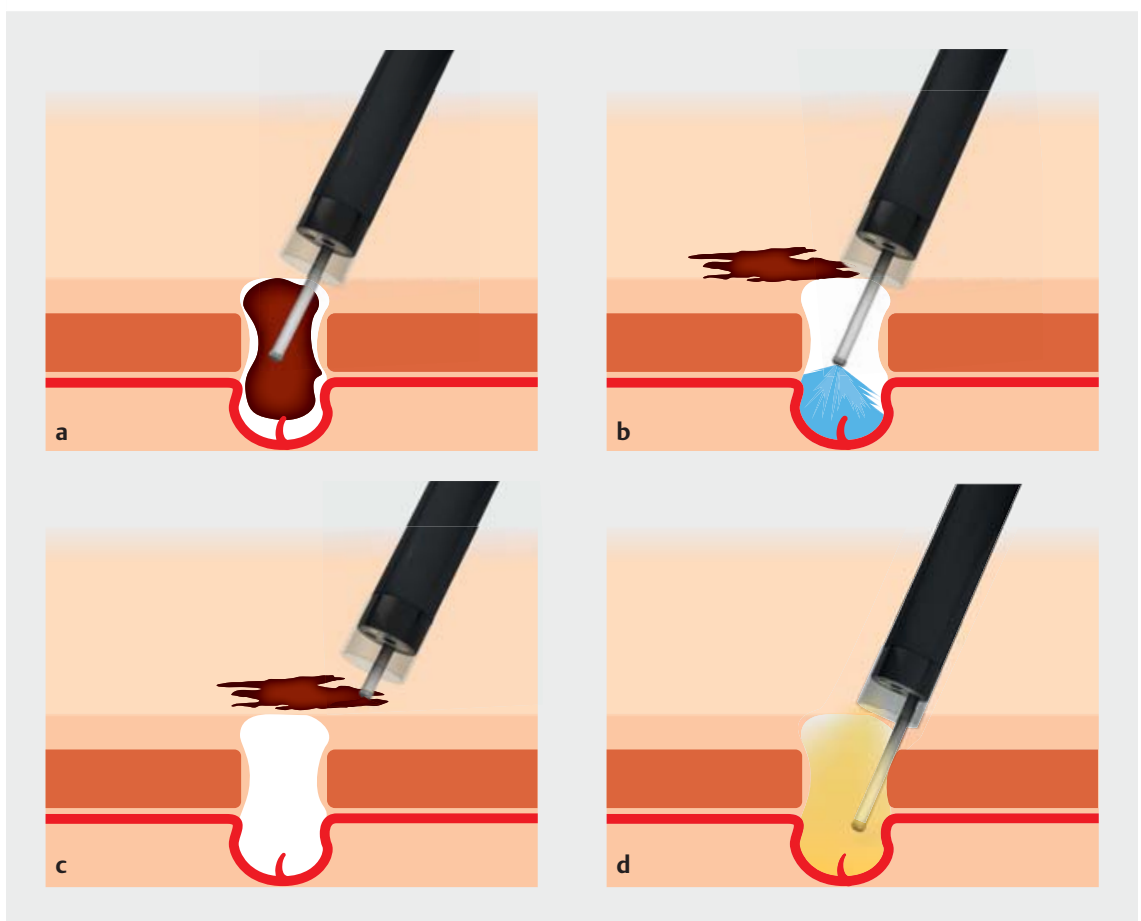

- Fig. 2 Schema of the nontraumatic tube method. a Insert the nontraumatic tube into the diverticulum where the adherent clot is attached. $\mathbf{b}$ Remove the blood clot by washing from the spray hole of the nontraumatic tube. c Remove the blood clot using suction from the spray hole of the nontraumatic tube. $\mathbf{d}$ Examine the diverticulum for signs of an exposed vessel by expanding the opening of the diverticulum using the nontraumatic tube.

the diverticulum opening, revealing an exposed blood vessel. Active bleeding was observed with further suctioning from the nontraumatic tube, indicating that this diverticulum was responsible for the hemorrhage ( $>$ Fig.3). Hemostasis was achieved using an over-the-scope clip at the diverticulum ( $\mathbf{F i g . 4 )}$ ). The patient was discharged without complications such as rebleeding or delayed perforation ( $>$ Video 1 ).

We believe that this novel nontraumatic tube method will contribute to the detection of blood vessels responsible for colonic diverticular hemorrhage and will facilitate the precise identification of an SRH.

Endoscopy_UCTN_Code_TTT_1AO_2AD

\section{Competing interests}

The authors declare that they have no conflict of interest.

The authors

Koichiro Kawano리 Mamoru Takenaka² Reiko Kawano', Daisuke Kagoshige ${ }^{1}$, Takao Kato ${ }^{1}$, Katsuhisa Nishi ${ }^{1}$, Masatoshi Kudo ${ }^{2}$

1 Department of Gastroenterology Hyogo Prefectural Awaji Medical Center, Sumoto, Hyogo, Japan

2 Departments of Gastroenterology and Hepatology, Kindai University Faculty of Medicine, Sayama, Osaka, Japan 

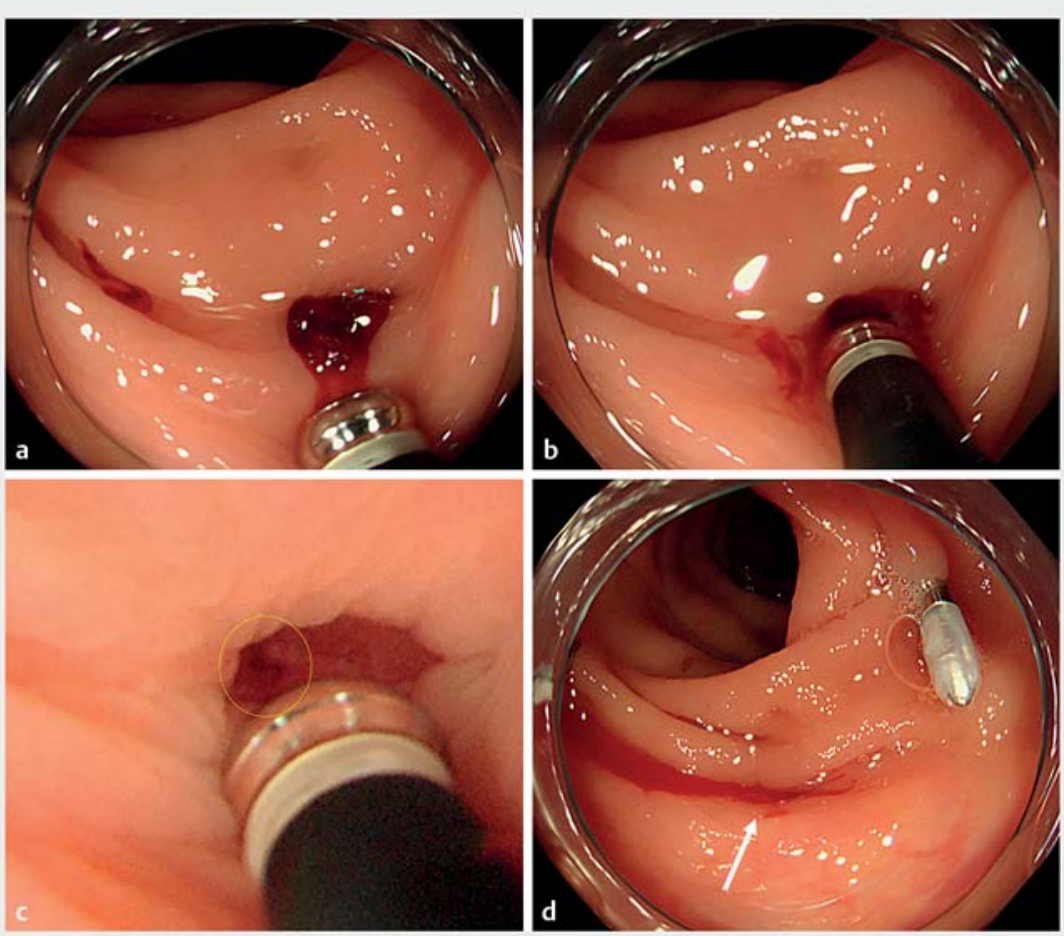

Fig. 3 Endoscopic images of the nontraumatic method. a Colonoscopy revealed an adherent clot attached to the diverticulum at the ascending colon. $\mathbf{b}$ A nontraumatic tube was inserted into the diverticulum, and the blood clot was removed by repeated washing and suction operations from the spray hole of the nontraumatic tube. $c$ By pressing the nontraumatic tube against the diverticulum wall and expanding the diverticulum opening, an exposed vessel (yellow circle) could be identified in the diverticulum. $\mathbf{d}$ Repeated suction at the exposed vessel caused an active bleed (arrow).
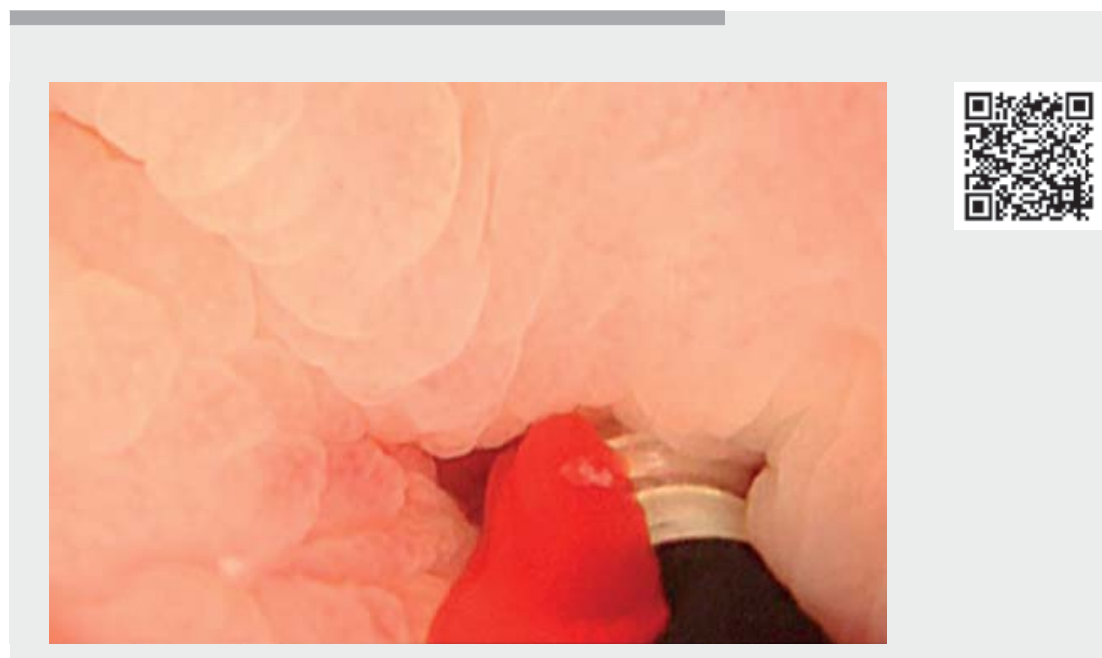

$\checkmark$ Video 1 The nontraumatic tube method is a new and useful method for the safe identification of a blood vessel responsible for colorectal diverticular hemorrhage.

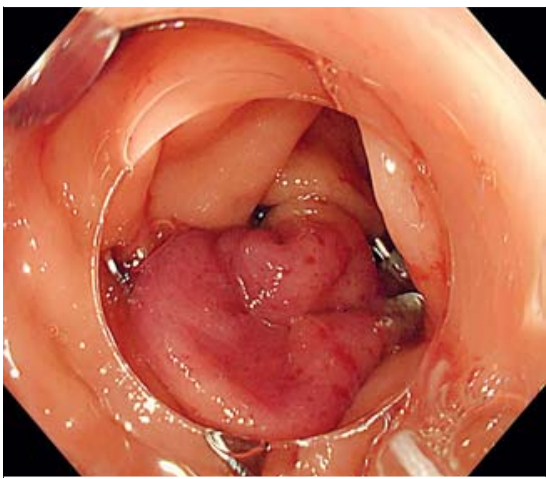

- Fig. 4 Hemostasis was achieved using an over-the-scope clip at the diverticulum.

\section{Corresponding author}

\section{Mamoru Takenaka, MD, PhD}

Department of Gastroenterology and Hepatology, Kindai University Faculty of Medicine, 377-2 Ohno-Higashi, Sayama, Osaka, 589-8511, Japan

mamoxyo45@gmail.com

\section{References}

[1] Burkitt DP, Walker AR, Painter NS. Effect of dietary fibre on stools and the transit-times, and its role in the causation of disease. Lancet 1972; 2: 1408-1412

[2] Kok KY, Kum CK, Goh PM. Colonoscopic evaluation of severe hematochezia in an Oriental population. Endoscopy 1998; 30 : 675-680

[3] Ohi G, Minowa K, Oyama T et al. Changes in dietary fiber intake among Japanese in the 20th century: a relationship to the prevalence of diverticular disease. Am J Clin Nutr 1983; 38: 115-121

[4] Jensen DM, Ohning GV, Kovacs TO et al. Natural history of definitive diverticular hemorrhage based on stigmata of recent hemorrhage and colonoscopic Doppler blood flow monitoring for risk stratification and definitive hemostasis. Gastrointest Endosc 2016; 83: 416-423

[5] Fujii T, Hasegawa RT, Saitoh Y et al. Chromoscopy during colonoscopy. Endoscopy 2001; 33: 1036-1041

\section{Bibliography}

Endoscopy 2022; 54: E240-E241

DOI $10.1055 / a-1486-6476$

ISSN $0013-726 \mathrm{X}$

published online 8.6.2021

(c) 2021. Thieme. All rights reserved.

Georg Thieme Verlag KG, Rüdigerstraße 14

70469 Stuttgart, Germany 